\title{
CARACTERIZACIÓN CUANTITATIVA DE LAS VARIABLES DE LA CULTURA ORGANIZACIONAL EN LA INSTITUCIÓN EDUCATIVA JOSÉ HOLGUÍN GARCÉS DE LA CIUDAD DE CALI- FEBRERO 2018
}

\author{
Emilse Cerón Tejada, Lady Johana Corral Caicedo, Erika Andrea Contreras Gamboa, \\ Carlos Lino Rengifo \\ Semillero de Investigación Gestión-E \\ Grupo de Investigación GICES \\ Institución Universitaria Antonio José Camacho
}

\section{RESUMEN}

La Cultura Organizacional ( $\mathrm{CO}$ ) ha tomado mayor relevancia en las últimas décadas, convirtiéndose en un elemento clave para el diseño de la estrategia corporativa, dado que en su esencia están contenidos los principios, creencias y valores que modelan los comportamientos y conductas que se presentan al interior de las organizaciones.

Considerando lo anterior, en esta investigación se presentan resultados que evidencian la importancia del modelo de Cameron \& Quinn y la aplicación de su herramienta OCAI, como uno de los instrumentos para identificar tipos de cultura en las organizaciones, en asociación con otras herramientas estadísticas, entre las cuales nombramos el Análisis de Componentes Principales (ACP), la Función VarImp y las redes neuronales, con el fin de lograr correlacionar los resultados en la identificación del tipo de cultura organizacional, predominante en la Institución Educativa José Holguín Garcés, permitiendo suministrar información con menor sesgo de subjetividad y proporcionando mayor grado de veracidad.

Es así como los resultados obtenidos de esta investigación reflejan mayor incidencia de la cultura de tipo Jerárquica en la institución educativa, sin dejar de mencionar que los tipos de cultura Clan, Mercado y Adhocrática también tienen gran representación, según los resultados arrojados en la investigación.

Adicionalmente, es necesario indicar que existe coherencia en los resultados obtenidos, tanto en la aplicación del OCAI, como de las herramientas APC y VarImp, donde la participación del tipo de cultura jerárquica presenta mayor nivel de predominancia.

\section{PALABRAS CLAVE}

Cultura Organizacional, Instrumento OCAI, APC (Análisis de Componentes Principales), Redes Neuronales, Función Varimp. 


\section{ABSTRACT}

The Organizational Culture (CO) has become more relevant in recent decades becoming a key element for the design of corporate strategy, since in its essence are contained the principles, beliefs and values that model the behaviors and behaviors that are presented to the inside of organizations.

Considering the above, this research presents results that demonstrate the importance of the Cameron \& Quinn model and the application of its OCAI tool, as one of the instruments to identify types of culture in organizations, in association with other statistical tools among the which we named the Principal Component Analysis (ACP), the VarImp function and the neural networks, in order to correlate the results in the identification of the type of organizational culture prevailing in the José Holguín Garcés Educational Institution, allowing to provide information with less bias of subjectivity and providing a greater degree of truthfulness.

This is how the results obtained from this research reflect a higher incidence of the hierarchical culture in the educational institution, not to mention that the types of Clan, Market and Adhocratic culture also have great representation, according to the results of the research.

Additionally, it is necessary to indicate that there is consistency in the results obtained both in the application of the OCAI, as well as in the APC and VarImp tools, where the participation of the type of hierarchical culture presents a higher level of predominance

\section{KEYWORDS}

Organizational Culture, OCAI Instrument, APC (Principal Component Analysis), Neural Networks, VarImp Function.

\section{INTRODUCCIÓN}

La naturaleza de las empresas y organizaciones está directamente ligada a los cambios y transformaciones que ocurren en su entorno y contexto, y uno de los aspectos más importantes en los cuales se afecta, es en su Cultura Organizacional.

Por esta razón es importante para las empresas identificar el tipo de cultura organizacional y las relaciones que se encuentran en su interior, dado la contribución que ésta puede proporcionar a la consecución de metas y objetivos planteados en el diseño de su estrategia corporativa, a través del aprovechamiento de los principios, creencias, valores y demás comportamientos que modelan su Cultura Organizacional.

Asimismo, es importante en el estudio sobre Cultura Organizacional (CO), entender como ésta no es estática e invariable y, por el contrario, tiene una naturaleza activa en cuanto a cómo es interpretada por los colaboradores al interior de las organizaciones en el cumplimiento de sus actividades diarias, esto basado en la necesidad imperante de alcanzar los objetivos y metas planteados por la organización. Así lo señala Vargas (2007): "Actualmente, el éxito competitivo está vinculado a la habilidad de la organización para lograr que sus activos intangibles, fundamentados en los conocimientos, habilidades, valores y actitudes de las personas, formen un núcleo central estable de la empresa" (p.145).

En este sentido y con la intención de identificar el tipo de CO para la Institución Educativa José 
Holguín Garcés, se ha escogido el modelo de "Valores en Competencia" de Cameron \& Quinn y su herramienta OCAI que permite establecer la predominancia de un tipo de $\mathrm{CO}$, entre los cuatro propuestos en su modelo (Clan, Adhocrática, Mercado y Jerárquica).

Adicionalmente, y con el propósito de obtener información más objetiva sobre la inclinación o prevalencia de un tipo o características de una $\mathrm{CO}$ sobresaliente, se ha optado por utilizar una técnica estadística denominada "Análisis de Componente Principales" (APC), la cual permite hacer una síntesis o reducción de la dimensión de la información, facilitando el proceso de análisis e inferencia sobre la misma. Para (Rodriguez Guerra I., 2004)

La Cultura tiene que ver con el proceso de socialización que se da dentro de una empresa, a través de una objetivación social. No existe organización sin una cultura inherente, que la identifique, la distinga y oriente su accionar y modos de hacer, rigiendo sus percepciones y la imagen que sus públicos tengan de ella. Las manifestaciones culturales son a su vez categorías de análisis, a través de las cuales se puede llevar a cabo el diagnóstico cultural en una empresa. (p.1).

Considerando la importancia que la cultura organizacional representa en una empresa y como parte de esta propuesta, se han identificado situaciones que tienen su origen en paradigmas, creencias y costumbres, alrededor de la gestión que se realiza al interior de la Institución Educativa José Holguín Garcés. Estas situaciones corresponden generalmente al modelo de dirección adoptado, en el que quien dirige estas instituciones, normalmente es una persona con formación profesional docente, dado que por dogma de este gremio de profesionales son ellos precisamente quienes tienen mayor conocimiento sobre los procesos, requerimientos y responsabilidad en la calidad educativa que se imparte. En opinión de : (Dávila, 1999):
La cultura organizacional es otro elemento clave para que los directores de las escuelas la consideren en los procesos de influencia y dirección. El trabajo del administrador, aquí, es hacer que los elementos culturales se manifiesten a través de aspectos simbólicos (ritos y creencias, historias y mitos, o lenguaje) con el mismo significado de las creencias básicas y valores centrales para que los administradores, maestros y estudiantes puedan compartirlos. (p.46).

El análisis de esta propuesta se realiza con la intención de identificar las variables cualitativas de la Cultura Organizacional de la Institución Educativa José Holguín Garcés de Cali, con el propósito de cuantificarlas y que puedan usarse a futuro como herramientas que contribuyan en la solución de su problemática interna y la toma de decisiones administrativas en pro de la mejora del servicio.

Los resultados de esta propuesta se basaron en el modelo de Cameron \& Quinn donde se analizan seis rasgos existentes al interior de las organizaciones para identificar el tipo de cultura predominante dentro de los cuatro que contempla su modelo (Clan, Adhocratica, Jerarquía y Mercado), enmarcados en dos dimensiones, la primera: "flexibilidad y discrecionalidad", "estabilidad y control", y la segunda: "orientación interna" y "orientación externa”. Para Cameron \& Quinn:

Este método es extremadamente útil para organizar e interpretar una amplia variedad de fenómenos organizacionales (...) También explicamos los cuatro tipos de cultura dominantes que emergen de este método. Estos cuatro tipos de cultura sirven como base para la OCAI. Además, porque la cultura define los valores fundamentales, supuestos, interpretaciones, y enfoques que caracterizan una organización, podríamos esperar que otras características de la organización también reflejaran los cuatro tipos de cultura. (2006, p. 31) 


\section{MARCO TEÓRICO}

\section{Cultura Organizacional}

Desde siempre la cultura ha sido "sinónimo" de las distintas expresiones de una sociedad, sus costumbres, rituales y valores que rigen su comportamiento, entre otros; es por ello que Schein citado por (Romo, 2008) la define como:

Un patrón de supuestos básicos compartidos que el grupo aprende en la medida que resuelve sus problemas de adaptación externa e integración interna, que los ha trabajado lo suficiente para ser considerados como válidos $y$, por lo tanto, dignos de ser enseñados a los nuevos miembros como la forma correcta de percibir, pensar y sentir en relación con esos problemas. (p. 24).

Considerando lo anterior, se puede inferir que el ámbito empresarial no es la excepción, dado que en éste también se presentan las expresiones de una sociedad $y$, por tanto, en él también hay una cultura implícita, y es precisamente esto último lo que ha motivado a tantos investigadores y a la academia en general, a estudiar las organizaciones desde la perspectiva de su cultura, a desarrollar modelos y teorías que permitan definir el tipo de cultura organizacional en éstas.

Teniendo en cuenta lo anterior y la necesidad de la búsqueda de información para esta investigación se hace indispensable la revisión bibliográfica de antecedentes, trabajos, investigaciones, artículos, libros y demás, realizados bajo el modelo de Cameron \& Quinn, que logran la identificación del tipo predominante de cultura organizacional de las empresas, además de mostrar la importancia que presenta la $\mathrm{CO}$ al interior de éstas, permitiendo tener una visión más clara y aproximada al objetivo general que se desea lograr. De esta forma, lo plantea (Vargas, 2007):

Es aquí donde cobra vigencia el estudio profundo de la cultura organizacional, pues se pretende, a partir de esta nueva tendencia, lograr que los empleados de la organización asuman actitudes que tradicionalmente la teoría organizacional, enfatiza, se dan, según sea la cultura "vivida en la empresa. (p.145).

Es así como con esta investigación se busca hacer la caracterización, identificación y análisis de la cultura organizacional en la Institución Educativa José Holguín Garcés de Cali, teniendo como componente principal la importancia que la cultura tiene al interior de toda organización, y que tanto estos valores, creencias, costumbres y demás rasgos hacen parte inherente de la identidad de las personas en su entorno laboral, como pueden identificarse ampliamente, en este caso, gracias al modelo de Cameron \& Quinn y su instrumento OCAI, adoptado para tal fin, encontrando que cada una de estas características cualitativas pueden llevarse a su cuantificación para una comprensión más objetiva, con ayuda de herramientas estadísticas que pueden llevar a una valoración, de modo que realizar el análisis de la información sea una tarea más cómoda.

Dicho lo anterior, el presente documento recoge algunos elementos de la cultura organizacional y permite la reflexión sobre los métodos y resultados obtenidos en referencia a CO. Para determinar el modelo de $\mathrm{CO}$ existen dos posibilidades, los enfoques cualitativos y los cuantitativos, la elección depende de los objetivos perseguidos, que pueden ser: caracterización, medición o intervención.

Los estudios cualitativos más comunes son los etnográficos, aunque no se deben desconocer otros como el Focus Group, la percepción de valores y filosofías; los juegos proyectivos, las entrevistas a profundidad y el Intercambio de roles, por mencionar algunos. De otra parte, puede afirmarse que los policymakers son precursores en la aplicación de enfoques cuantitativos para la medición de la CO. Muchos de ellos han validado instrumentos en el ámbito gubernamentalhospitalario y educativo-, usando como base fundamental el Modelo de los Valores en Competencia, de Cameron \& Quinn. (Torres \& Rodriguez, 2009) 
Como se puede apreciar, existen diferentes métodos y herramientas para medir y/o determinar el tipo de $\mathrm{CO}$, siendo la caracterización y medición del impacto de ésta, de gran importancia en la obtención de resultados directos y la eficiencia que pueda aportarle a la organización.

En el documento anteriormente mencionado, se encontró otra importante conclusión que aporta al desarrollo de este estado de arte, una valiosa incógnita al cuestionarse si quien define el carácter de la organización es su gente o la CO de la misma:

Las definiciones de $\mathrm{CO}$ además de numerosas, resultan complejas y divergentes; todos saben qué es, pero pocos son capaces de identificarla y explicarla. Más aún, muchos son los que la invocan, especialmente en el medio latinoamericano, y pocos quienes realizan propuestas concretas que permitan su caracterización y medición. Si bien las definiciones son variadas, todas reflejan unos elementos comunes como: mandatos aprendidos, reglas no escritas, supuestos compartidos, valores y prácticas compartidas, conducta aceptable, modo correcto y un moldear de la conducta humana. Conceptos que en conjunto evidencian la informalidad compartida que gobierna y legitima la conducta humana. En muchas organizaciones, incluidas las entidades gubernamentales, la preocupación actual se concentra en el personal, al considerarlo el activo más valioso, apreciación un tanto desfasada, por cuanto lo que verdaderamente define el carácter de toda organización es su cultura y no su gente. (Torres \& Rodriguez, 2009, pág. 131)

Es así que los autores se concentran en la caracterización y medición de la $\mathrm{CO}$, indicando en su documento que ésta proviene y es forjada por la organización como tal, siendo cada colaborador de ella, quien la va adoptando y la va exteriorizando con sus conductas, consiguiendo así mejorar la acción colectiva y el desempeño social.
En cuanto a cuál es el objetivo de identificación de la CO en las empresas, con respecto a la práctica de valores, compromiso y trabajo en equipo para el logro de una mejora en la eficiencia productiva y mayor competitividad, se puede ver en el siguiente estudio sobre la relación existente entre la cultura organizacional de la empresa y su rendimiento, donde se infiere que:

Los resultados de este estudio confirman los de otras investigaciones, ya que muestran que los valores y prácticas organizacionales, asociadas a la cultura de innovación, es decir, aquellas que estimulan la cohesión, el trabajo en equipo, el compromiso, la adaptabilidad, la flexibilidad, la aceptación del riesgo y la incertidumbre, generan importantes mejoras en el desempeño, en términos de la calidad de los productos y servicios, en la eficiencia de los procesos internos, en las relaciones humanas internas, y en la relación de la empresa con su entorno inmediato. Este resultado evidencia la sinergia que se genera al combinar adecuadamente prácticas de la cultura clan y de la cultura adhocrática. (Albarracín \& Lema, 2011, pág. 141)

Con respecto a lo indicado por los autores Albarracín y Lema en la anterior cita, se puede reafirmar que las buenas prácticas en las organizaciones, flexibilidad, adaptación y combinación de diferentes tipos de cultura, que generen en los colaboradores estímulos positivos en sus relaciones interpersonales y laborales pueden coadyuvar al crecimiento y fortalecimiento de la organización.

Continuando con este análisis, se encontró el artículo Cultura organizacional y organización que aprende, que tiene como objetivo de trabajo analizar la importancia del diagnóstico de la $\mathrm{CO}$ en la empresa, como condición que promueve la capacidad de innovación de las personas y de la propia organización, concluyendo: 
Con esta investigación se validan los instrumentos para caracterizar la Cultura Organizacional y la capacidad de aprendizaje y se ofrece información que confirma la necesidad de ampliar y profundizar los estudios de la cultura, organización y el aprendizaje organizacional, con diferentes enfoques, técnicas y métodos, que permita un acercamiento más objetivo a la realidad, por su importancia en la innovación y la creación de ventajas competitivas. Por ello, profundizar en los factores que obstaculizan y favorecen la innovación desde los factores internos a la organización, sin obviar los externos, es una tarea que requiere del esfuerzo continuado de las investigaciones en la región. (Acosta, Ramos, Rio, \& Morejon, 2012, pág. 47)

Con lo anterior, se ratifica la importancia del estudio de la CO, en este caso, desde la perspectiva de la innovación y haciendo énfasis en el uso de diferentes métodos y técnicas que permitan un acercamiento más objetivo hacia los resultados obtenidos.

\section{Modelo de Cameron \& Quinn}

Este modelo propone el análisis de seis rasgos existentes al interior de las organizaciones para identificar el tipo de cultura predominante dentro de los cuatro que contempla su modelo (Clan, Adhocracía, Jerarquía y Mercado), enmarcados en dos dimensiones, la primera: "flexibilidad y discrecionalidad" y "estabilidad y control", y la segunda: "orientación interna" y "orientación externa”.

Este método es extremadamente útil para organizar e interpretar una amplia variedad de fenómenos organizacionales (...) También explicamos los cuatro tipos de cultura dominantes que emergen de este método. Estos cuatro tipos de cultura sirven como base para la OCAI. Además, porque la cultura define los valores fundamentales, supuestos, interpretaciones y enfoques que caracterizan una organización, podríamos esperar que otras características de la organización también reflejaran los cuatro tipos de cultura. (Cameron \& Quinn, 2006, p. 31).

Dado que la investigación y los resultados del presente documento están basados en el modelo de Cameron \& Quinn, se hace claridad sobre el instrumento usado para tal fin: el OCAI (Organizational Culture Assessment Instrument), que en palabras de sus autores es: Un instrumento que permite diagnosticar la orientación dominante de su propia organización basada en los tipos de cultura básicos. También ayuda en el diagnóstico de la intensidad de su cultura organizacional, tipo de cultura y congruencia. (Cameron \& Quinn, 2006, p 37).

\section{Análisis de Componentes Principales (PCA)}

Con los datos resultantes en la aplicación del cuestionario OCAI, se realiza un segundo análisis usando como instrumento la herramienta estadística PCA, con lo que se pretende hacer una evaluación multivariada de carácter cuantitativo sobre las variables objeto de estudio. Esta es una técnica utilizada para reducir la dimensión del conjunto de datos, y de esta manera, hacer que la información sea más comprensible y cómoda para su manejo y las observaciones que permitan hacer inferencias, así como conclusiones.

Esta técnica de análisis de datos es frecuentemente usada en análisis de datos permite medir correlaciones clave para un conjunto de indicadores, muestra la dirección de las correlaciones, y resume los diversos indicadores en un número limitado de factores interpretables. (Olaf Merk, 2013, pág. 9)

\section{Redes Neuronales}

En los últimos años, las redes neuronales han sido aplicadas a diversos campos como herramienta para solucionar problemas dados, por su capacidad de combinar parámetros que permiten emular y predecir patrones de comportamiento. 
De las RN como son conocidas, se puede decir que son modelos inspirados en la neurociencia y que han sido influenciados con métodos estadísticos, así como por la teoría general de los sistemas, por mencionar algunos campos de conocimiento.

Las redes neuronales artificiales (RNA) o sistemas conexionistas son sistemas de procesamiento de información, cuya estructura y funcionamiento están inspirados en las redes neuronales biológicas. Consisten en un conjunto de elementos simples de procesamiento llamados nodos o neuronas conectadas entre sí por conexiones que tienen un valor numérico modificable, llamado peso (Moreno, 2002, pág. 17).

Con estos pesos se calcula el producto de las ponderaciones de la estructura interna de la red neuronal, al final la suma de estos productos en todas las neuronas ocultas definirá la variable de importancia (Olden, 2004, pág. 8).

Como resultado, este proyecto pretende aportar al sector académico y empresarial un instrumento de clasificación, que logre eliminar la subjetividad actual en la valoración de la $\mathrm{CO}$ en las empresas, de esta manera, la toma de decisiones frente a las problemáticas actuales tendría un análisis más objetivo y confiable en comparación con los métodos de valoración que existen hoy en día para este componente del Desarrollo Organizacional.

\section{METODOLOGÍA}

Para el desarrollo del proyecto se tienen en cuenta dos tipos de estudio:

Descriptivo: se pretende describir las variables de la cultura organizacional a partir del modelo de Cameron \& Quinn.

Exploratorio: de acuerdo con las dimensiones planteadas por Cameron \& Quinn se pretende determinar cuál es el tipo de cultura organizacional que caracteriza la empresa.
En cuanto a los métodos de Investigación, se plantean los siguientes: Observación: a través del estado del arte, se identifican las distintas variables que conforman la cultura organizacional.

Analítico: se busca distinguir y separar los componentes e indicadores que van a definir las variables cuantificables de la cultura organizacional.

Deductivo: a partir de todos los aspectos cuantificables, determinar el tipo de cultura organizacional.

\section{Fuentes y técnicas de recopilación de la información}

Fuentes primarias: se realizarán encuestas para realizar una prueba piloto y finalmente utilizar la herramienta OCAI (Organizational Culture Assesment Instrument).

Fuentes secundarias: se emplearán referentes bibliográficos, la consulta de textos e información especializada para los procesos de análisis correspondientes:

Identificación de las variables de la cultura organizacional, observación y análisis documental.

Población: en particular para este proyecto de investigación, la población objeto de estudio, está conformada por 123 personas, entre docentes, directivos, docentes y personal administrativo que labora actualmente en la Institución Educativa José Holguín Garcés de la ciudad de Santiago de Cali.

\section{Esquema metodológico}

Objetivo 1. Método de investigación: observación y análisis, ampliación del estado del arte, los instrumentos de medición de la Cultura Organizacional; construcción de la herramienta, a través de los indicadores encontrados y validación de los indicadores, mediante una prueba piloto. 
Objetivo 2. Método de investigación: análisis, parametrización estadística, análisis exploratorio de datos: aproximación estadística de las variables cuantificables, aproximación paramétrica de los indicadores cuantificables y análisis exploratorio de las variables cuantificables.

Objetivo 3. Método de investigación: análisis y deducción, determinar el tipo de cultura organizacional, herramienta OCAI, aplicación de la herramienta OCAI y contrastar los resultados con el método clásico de Cameron \& Quinn.

\section{MUESTRA}

En la búsqueda de una muestra idónea que arroje la mejor calidad de información para este proyecto, se optó por el cálculo de muestras en poblaciones finitas, con lo que se pretende obtener el número de cuestionarios, a aplicar entre los colaboradores de la Institución Educativa José Holguín Garcés.

$$
\mathrm{n}=\frac{\mathrm{N}(\mathrm{Z})^{2}(\mathrm{p})(\mathrm{q})}{(\mathrm{d})^{2}(\mathrm{~N}-1)+(\mathrm{Z})^{2}(\mathrm{p})}
$$

Para ilustrar mejor lo anteriormente descrito, se presenta fórmula y cálculo de la respectiva muestra:

$$
\mathrm{n}=\quad \begin{gathered}
123(1,96)^{2}(0,5)(0,5) \\
(0,06)^{2}(123-1)+(1,96)^{2}(0,5)(0,5)
\end{gathered}
$$

\section{ANÁLISIS DE RESULTADOS}

\section{Resultados OCAI}

Los resultados que se reflejan a continuación corresponden a la sumatoria de las valoraciones que dieron las personas participantes en la aplicación del OCAI, a cada uno de los subfactores contemplados en el cuestionario.
Tabla 1. Análisis de la situación actual

\begin{tabular}{|c|c|c|c|}
\hline Clan (A) & Adhocrática (B) & Mercado (C) & Jerárquica (D) \\
\hline 1435 & 975 & 1166 & 1462 \\
\hline
\end{tabular}

Como se puede apreciar en la gráfica, la Institución Educativa José Holguín Garcés posee una mezcla de características que dejan ver cómo cada uno de los tipos de cultura identificados en el modelo de Cameron \& Quinn tiene un buen porcentaje de participación. Si bien es cierto la información arroja un mayor peso sobre la cultura Jerárquica, con los resultados no es posible identificar clara o definitivamente la predominancia de un sólo tipo de cultura especifico.

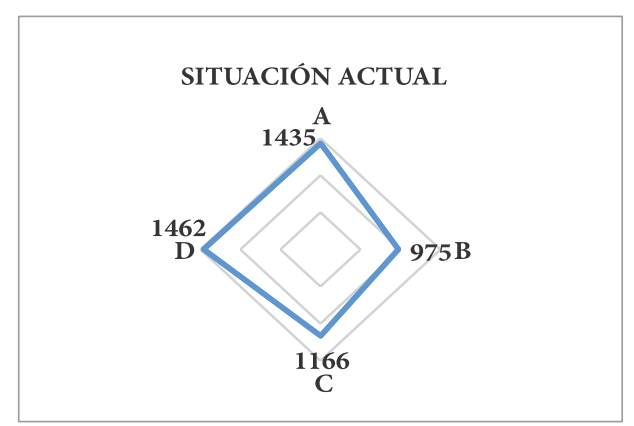

Fig.1 Expresión gráfica datos definidos situación actual. Fuente: elaboración de los autores.

Es así como con una mayor prevalencia, las culturas Jerárquica y Clan sobresalen, teniendo un mayor porcentaje de participación en los resultados obtenidos, indicando esto que la Institución Educativa José Holguín Garcés es un tipo de organización o lugar de trabajo estructurado, guiado por procedimientos y procesos bien definidos, normas, reglas; así como también cuenta con buenos líderes, estabilidad laboral, y políticas que la sostienen como una buena institución. Adicional a esto, los colaboradores de la institución se sienten como en familia, en un lugar de trabajo amigable y cómodo para laborar; la colaboración, lealtad y la tradición son valores que pueden llevarlos al éxito. 


\section{Resultados PCA}

Gráfico 1. Componentes principales

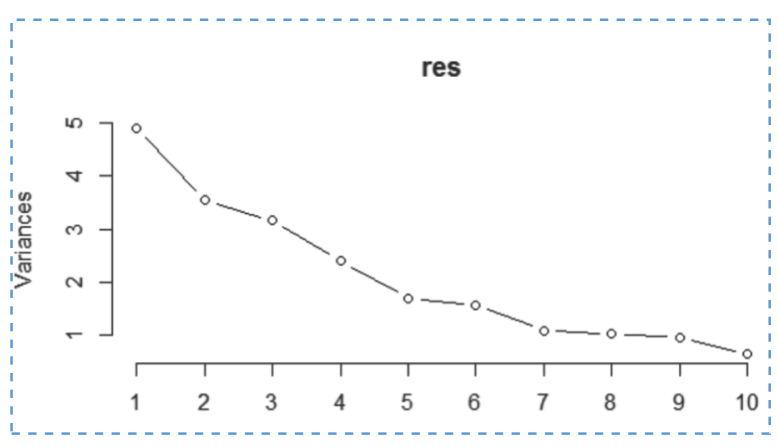

Fuente: elaboración de los autores.

Con respecto a la ilustración anterior, se puede observar que la mayor proporción de información se encuentra concentrada de manera mayoritaria en los primeros seis (6) componentes, por lo que a partir de este momento el análisis de la información se centrará en éstos.

\section{Resultados Redes Neuronales}

Tabla 2. Importancia de los subfactores

\section{Variables}

Overall

D3 6.462

D5 6.321

C6 6.155

D6 6.068

A4 5.683

A5 5.570

C3 5.205

A6 4.978

C1 4.766

A1 4.757

A2 4.644

B3 4.416

D1 4.288

C5 4.127

B2 4.017

D2 3.511

B4 3.401

C2 3.143

B1 2.989

B5 2.455

Fuente: PCA
Los resultados obtenidos al aplicar redes neuronales para encontrar las variables más importantes de los datos que arroja el OCAI, permiten observar que en general los subfactores de mayor concentración de datos son:

(D3 - Administración del talento humano): el estilo de manejo del recurso humano se caracteriza por la implantación de normas y guías para la ejecución de procesos.

(D5 - Énfasis estratégico): gestión interna con énfasis en el cumplimiento eficaz y eficiente de la misión y los objetivos de la institución.

(C6 - Criterio de éxito): la prestación de sus servicios se centra en la calidad y el liderazgo, como clave para el sector educativo.

(D6 - Criterio de éxito): el éxito de la institución se liga al cumplimiento y consecución eficiente de sus tareas.

(A4 - Adhesión a la organización): el compromiso, lealtad y confianza son pilares de pertenencia a la institución.

Con la información obtenida con redes neuronales, se puede observar que las variables de mayor importancia confirman que el tipo de cultura organizacional predominante en la Institución Educativa José Holguín Garcés es la Jerárquica, donde los controles, normas, políticas, protocolo, hacen parte inherente a su cultura.

Se observa además en los resultados el criterio de éxito para la institución educativa presenta proporción semejante en los tipos de cultura Jerárquica y Mercado, evidenciando esto que el liderazgo en el mercado y cumplimiento de tareas hacen parte de sus prioridades. 


\section{CONCLUSIONES}

Con base en los resultados obtenidos en la aplicación del cuestionario OCAI en la Institución Educativa José Holguín Garcés, se evidencia que existe una tendencia hacia el tipo de cultura Jerárquica; sin embargo, la cultura de tipo Clan, también cuenta con gran participación en el resultado obtenido, siendo ésta, segunda en orden de predominancia. Asimismo, estos resultados muestran gran representación de los tipos de cultura Adhocrática y Mercado.

Considerando las observaciones anteriormente descritas, se evidencia que coexisten al interior de la institución educativa los cuatro tipos de $\mathrm{CO}$, y por su naturaleza como prestadora de servicios esenciales de carácter público, prima su condición burocrática que la llevan a ejecutar sus actividades, regidas por políticas, normas, controles, reglamentos y eficiencia, propios de la cultura Jerárquica, así como también comportamientos de cordialidad, amistad, ambiente colaborativo, competencia, innovación y creatividad, entre otros, que caracterizan a los tipos de cultura Clan, Adhocrática y Mercado.

En relación con el análisis de la información realizado con PCA, se pudo observar que el mayor impacto en los resultados obtenidos, fue generado por los primeros seis (6) componentes principales, los cuales contienen la mayor proporción de datos.

Por otra parte, en el proceso de medición de la importancia que representan los subfactores para las personas que participaron en el desarrollo del cuestionario (usando la herramienta PCA), se pudo determinar que como consecuencia de que la cultura organizacional de la institución educativa tiene una mezcla de los cuatro tipos de $\mathrm{CO}$ al interior de ésta, con proporción igual entre los valores resultantes de sus subfactores, se hace necesaria la extracción de aquellos resultados individuales en que dos o más de los subfactores presentasen un mismo valor.

Considerando lo anterior, se hace necesario indicar que si bien el resultado obtenido en la medición de importancia de los subfactores está sesgado, refleja algo de coherencia con los resultados generales obtenidos en la aplicación del OCAI, donde la participación del tipo de cultura Jerárquica presenta alto nivel de predominancia.

\section{REFERENCIAS BIBLIOGRÁFICAS}

Acar, A. Z., \& Acar, P. (2014). Organizational Culture Types and Their Effects on Organizational Performance in Turkish Hospitals. Emerging Markets Journal.

Acar, A. Z., \& Acar, P. (2014). Organizational Culture Types and Their Effects on Organizational Performance in Turkish Hospitals. Emerging Markets Journal.

Acosta, M. d., Ramos, L. G., Rio, F. G., \& Morejon, V. M. (2012). Cultura organizacional y organizacion que aprende un analisis de la perspectiva de la innovacion. Coahuila, Mexico.

Albarracín, E. J., \& Lema, D. G. (2011). Cultura organizacional y rendimiento de las Mipymes de mediana y alta tecnología: un estudio. Cuadernos de administración, 141.

Anonimo. (2002). Cultura organizcional. Revista de Medicina IMSS 2002, 203 - 2011.

Anónimo. (2009). Universidad de Granada. Obtenido de http://www.ugr.es/ rescate /practicum/el_m_todo_de_observaci_n.htm

Arias, F. G. (2006). El Proyecto de Investigación Introducción a la Metodología Científica (Cinco ed.). Caracas, Venezuela: Texto C.A.

Cameron, K. S., \& Quinn, R. E. (2006). Diagnosing and Changing Organizational Culture. San Francisco, United Stated of America: John Wiley \&Sons, Inc. 
Castrillon, S. P. (2014). Caracteristicas predominantes de la cultura organizacional de la fuerza de ventas de la empresa SUSUERTE S.A. en el municipio de Manizales. Manizalez, Caldas, Colombia.

Constitución Política de Colombia. (1991). Constitución Política de Colombia. Constitución Política de Colombia. Bogotá.

Dávila, A. (1999). Cultura organizacional en una escuela católica mexicana: un estudio de caso. México: Siglo XXI Editores.

El Congreso de la República de Colombia. (21 de 12 de 2001). Ley 715 de Diciembre 21 de 2001. Ley 715 de Diciembre 21 de 2001. Bogotá.

Galvez Albarragan Edgar Julian, D. G. (Enero - Junio de 2011). Cultura organizacional y rendimiento de las Mypimes de mediana y alta tecnologia: un estudio emperico en Cali Colombia. Bogota.

Guerra, I. R. (01 de 10 de 2004). Gerencie.com. Obtenido de http://www.gerencie.com/culturaorganizacional.html

Ibarra, C. (26 de 10 de 2011). Metodologia de la Investigacion, blogstop. Obtenido de http://metodologadelainvestigacinsiis.blogspot.c om.co/2011/10/tipos-de-investigacionexploratoria.html

Icontec. (s.f.). Icontec Internacional. Recuperado el 10 de 2015, de http://www.acreditacionensalud. org.co/acreditacion.php? IdSub=115\&IdCat=29

Kim S. Cameron, R. E. (2006). Diagnosing and Changing Organizational Culture: based on the competing values framework. San Francisco, United States of America: John Wiley \& Sons, Inc.

Kim S. Cameron, R. E. (2006). Diagnosing and Changing Organizational Culture: based on the competing values framework. San Francisco, United States of America: John Wiley \& Sons, Inc.

Kuhn, M. (Noviembre de 2008). Building Predictive Models in R Using the Caret Package. Journal of Statistical Software, 28, 17, 19.

Laurence, F. S. (2004). El modelo Competing Values Framework (CVF) y el diagnóstico de la cultura organizacional. Economía y Administración, 2021.

Laurence, F. S. (Diciembre de 2004). El Modelo Competing Values Framework (CVF) y el Diagnóstico de la Cultura Organizacional. ECONOMÍA Y ADMINISTRACIÓN (63), 20.

Ministerio de Educación Nacional. (05 de 2009). Organización del Sistema Educativo. Organización del Sistema Educativo. Bogotá.

Moreno, J. J. (2002). Redes Neuronales Artificiales aplicada al Análisis de Datos. Redes Neuronales Artificiales aplicada al Análisis de Datos. Palma de Mallorca, España.

Olaf Merk, T.-T. D. (16 de 12 de 2013). The Effectiveness of Port-City Policies: A Comparative Approach. Recuperado el 14 de 05 de 2016, de http://dx.doi.org/10.1787/5k3ttg8z n1zt-en: http://www.oecd-ilibrary.org/urban -rural-and-regional-development/theeffective ness-of-port-city-policies_5k3ttg8zn1zt-en

Olden, J. (2004). An accurate comparison of methods for quantifying variable importance in artificial neural networks using simulated data. Ecological Modelling, (February). https://doi.org/10.1016/S0304-3800(04)00156-5

Ouchi, W. (1984). Teoría Z. Barcelona: Ediciones Orbis S.A. 
Raisirys González, F. S. (2008). Aspectos básicos del estudio de muestra y población para la elaboración de los proyectos de investigación. Universidad de Oriente Núcleo de Sucre, Escuela de Administración curso especial de grado, Cumaná.

Ramon, R. (2006). Historia y Evolución del Pensamiento Cientifico. Mexico.

Raquel Florez López, J. M. (2008). Las Redes Neuronales Artificiales Fundamentos Teóricos y Aplicaciones Prácitcas. La Coruña: Netbiblo, S.L.

Roberto Hernande Sampieri, S. M. (14 de 06 de 2012). Construccion de un instrumento para medir el clima organizacional en funcion del modelo de los valore en competencia. Construccion de un instrumento para medir el clima organizacional en funcion del modelo de los valore en competencia. Ciudad de Mexico, Mexico.

Rodriguez Guerra I. (2004). Cultura Organizacional. Cuba: Universidad de la Habana.

\section{AUTORES}

Erika Andrea Contreras Gamboa: Magíster en Gerencia del Talento Humano (Universidad de Manizales), Especialista en Alta Gerencia, Administradora de Empresas (Universidad de Pamplona). Coordinadora del Semillero de Investigación Gestión-E, Docente Tiempo Completo Facultad de Ciencias Empresariales en Institución Universitaria Antonio José Camacho. Grupo de Investigación - GICES. Email: econtreras@admon.uniajc.edu.co

Lady Johana Corral Caicedo: Egresada en Administración de Empresas Facultad de Ciencias Empresariales. Semillero de Investigación Gestión-
Romo, R. S. (2008). Emprendurismo, cultura,clima y comunicación. Málaga, España: Grupo Eumednet.

Sampieri, R. H., Valencia, S. M., \& Soto, R. C. (2012). Construcción de un instrumento para medir el clima organizacional en función del modelo de los valores en competencia. Contaduría y Administración (59), 237.

Schein, E. H. (2004). Organizational Culture and Leadership (3rd Edition ed.). San Francisco, United States of America: John Wiley \& Sons, Inc.

Sun, S. (2008). Organizational Culture and Its Themes. International Journal of Business and Management, 3(12), 137.

Torres, C. E., \& Rodriguez, J. C. (2009). Asuntos teoricos y metodologicos de la cultura organizacional. Mexico: Civilizar 9.

Vargas, O. H. (Marzo de 2007). La cultura humana y su interpretación desde la perspectiva de la cultura organizacional. Pensamiento y Gestión (No. 22), 145.

E, Facultad de Ciencias Empresariales en Institución Universitaria Antonio José Camacho. Email: lj.cc@hotmail.com

Emilse Cerón Tejada: Egresada en Administración de Empresas. Semillero de Investigación Gestión-E, Facultad de Ciencias Empresariales en Institución Universitaria Antonio José Camacho. Email: emicert@hotmail.es

Carlos Lino Rengifo: Ingeniero Electrónico de la Universidad Nacional de Colombia, Candidato a Magister en Ingeniería Universidad del Valle, Docente Tiempo Completo Facultad de Ingenierías Institución Universitaria Antonio José Camacho. Email:clino@admon.uniajc.edu.co 